\title{
Analytical modelling of composite panels subjected to impact loading
}

\author{
C. Navarro, J. Rodríguez* and R. Cortés* \\ Department of Engineering, Carlos III University, Avda. de Butarque 15, Leganés, 28911 Madrid, Spain \\ * Department of Materials Science, Polytechnic University of Madrid, E.T.SI. de Caminos, Canales y \\ Puertos, Ciudad Universitaria s/n, 28040 Madrid, Spain
}

\begin{abstract}
Résumé. Dans cet article nous presentons un modèle analitique pour étudier le comportement des materiaux composites. Dépendant de l'architecture des fibres, deux classes de materiaux sont envisagés: Composites a fibres unidirectionelles, et composites avec les fibres tissés. On ne considère que des projectiles de petit calibre. Suivant l'étude de l'impact sur une seule fibre, on présente un modèle simplifié: Puis le modèle est étendu pour tenir compte de l'architecture des fibres et de l'effet de la matrice. Les prédictions du modèle sont comparées avec des resultats expérimentaux ce qui illustre les possibilités du modèle.
\end{abstract}

\begin{abstract}
This paper deals with the analytical modelling of fibre-reinforced polymeric matrix composites under impact loading. Two types of composites are considered in this paper depending on their fibre architecture: unidirectional fibres and woven fabric composites. The impacting projectiles involved correspond to low caliber ammunition. After studying the impact behaviour of a single fibre, a simplified impact model is presented. The model is then extended to incorporate the fibre architecture and the matrix effects. Finally, the results predicted by the model are compared with experimental evidence, illustrating the model possibilities.
\end{abstract}

\section{INTRODUCTION}

Analytical models for impact problems become a very important tool when designing structural protection against impact. This is due to the fact that they allow a large number of parametric analyses of practical situations to be carried out, saving computer time and thus optimizing the final design of ballistic armours. Composite materials have shown a great efficiency in impact applications both as protective panels and as backing plates for other materials (such as ceramic materials). Despite the importance of composite materials in ballistic applications, their dynamic characterization at high strain rates is not well understood and it is now a hot point: manufacturers usually give only static properties of fibres and resin or static properties of the resultant composite. Much effort has been devoted in the past to study composites response in the low velocity regime of impact. Under high velocity impact conditions, the structural response of a composite panel is less important than in the case of low velocity impact: the damage area is now localized a few centimeters around the stagnation point and thus the geometrical characteristics of the panel become less important. Before describing simplified models for impact problems involving composite materials, the behaviour of a single fibre, when it is transversely impacted by a projectile, is briefly analyzed, making a particular emphasis on the type of travelling waves that appear in such a problem. From this study, a very simplified engineering model can be developed for unidirectional composites, calculating the defeating force applied by the fibres on the projectile, and then applying Newton's second law to the impacting body in order to obtain its deceleration history. Several composite properties affecting its impact response, such as fibre properties (fibre volume fraction, elastic modulus and failure strain), matrix resin properties, composite areal density, etc., are also implemented into the model and the way of doing this is discussed. In the proposed model a simplified solution to account for the effect of the matrix content, 
based on the assumption that the influence of the matrix has only an inertial character, is chosen. Before extending the above-mentioned model to woven fabric composites, the behaviour of simple fabric panels, without any resin content, is investigated. In the present model, the kinetic energy of the impacting body is mainly absorbed in the form of elastic and kinetic energy in the fibres directly in contact with the impacting body. The interaction effects between warps and filling fibres at crossovers complicates the wave propagation in the fabric and approximate solutions for such effects are included in the model for fabric impact response. Subsequently, the effect of resin content is also incorporated in the model.

For all the proposed analytical models, their predictions in several impact problems are compared with experimental results that appear in the open literature, which shows the model capability to solve these kinds of problems. In particular, experimental ballistic impact results on polyethylene fabric/polyethylene matrix, kevlar fabric/polyester resin, etc., will be considered.

\section{DEVELOPMENT OF THE SIMPLIFIED MODEL}

\subsection{Model for a single fibre subject to transverse impact}

This problem has been broadly investigated in the past, because the dynamic properties of a single fibre may be obtained directly by comparing the experimental results with those computed using the wellknown one-dimensional wave propagation theory. One of the first attempts to model fibre behaviour under lateral impact loading was carried out by Smith (1), who solved the problem by applying one-dimensional elastic wave theory. When a fibre is laterally impacted by a projectile two travelling waves start to propagate (see Figure 1) away from of the stagnation point.

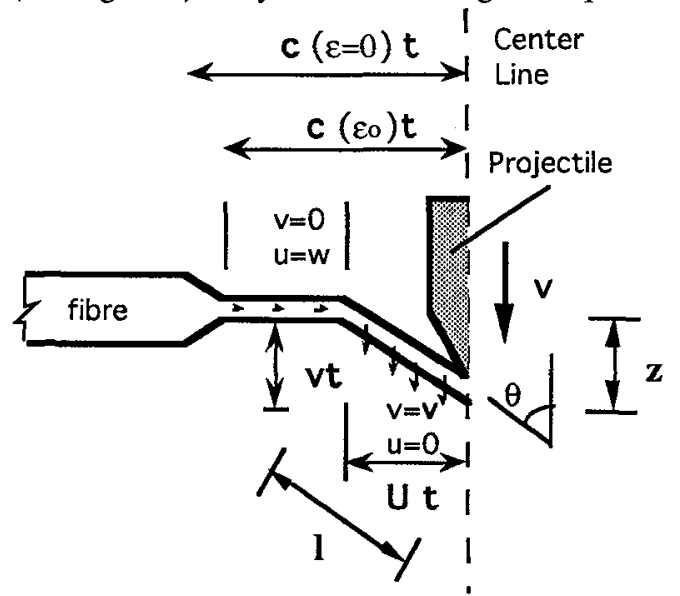

Figure 1
The faster one is a tensile longitudinal wave that propagates at the sound speed of the fibre material (c), which usually depends on the fibre strain (e), if an elastic non-linear material behaviour is assumed. The second wave is a transverse wave travelling at a speed $U$, with respect to an observer placed in the laboratory, which is a function of the stress $s$, strain e and density $\rho$ of the fibre. This last wave causes V-shaped pattern in the fibre, which was initially horizontal. Its propagation velocity, which is lower than that of the tensile longitudinal wave, is given by:

$$
\mathbf{U}=\sqrt{\frac{\mathbf{s}}{\rho(1+e)}}
$$

When a fibre particle has been traversed by the longitudinal tension wave, it moves towards the point of impact, with a horizontal velocity $w$ equal to the product of $\mathbf{c}$ by $\mathbf{e}$.

When a fibre particle is reached by the transverse wave front, it experiences an abrupt change in its motion, changing from moving horizontally at the velocity $\mathbf{w}$, to move transversely to the fibre, at the projectile velocity $\mathbf{v}$. All these magnitudes (c,e, $\mathbf{U}, \mathbf{w}$ and $\mathbf{v}$ ) are related to one another as may be seen in Smith's article [1]. There are experimental observations on the behaviour of a transversely impacted single fibre. So, Petterson et al. [2] have shown that fibre induced strain decays softly from a maximum value, at the impact point, to zero at the tension wave front location. They considered nylon fibres and impact velocities ranging from 229 to $238 \mathrm{~m} / \mathrm{s}$. The force $\mathbf{F}$, opposing to the projectile advance, may be calculated as:

$$
\mathbf{F}=2 \mathbf{s} \mathbf{S}_{\mathrm{f}} \cos \mathbf{q}
$$

where $\mathbf{s}$ is the normal stress acting on the fibre cross-section, $\mathbf{S}_{\mathbf{f}}$ the fibre cross-sectional area and $\mathbf{q}$ the angle formed by the part of the fibre affected by the transverse wave propagation and the projectile direction. If the fibre material had a linear-elastic behaviour, the fibre stress could be computed directly as the product of the fibre's Young modulus $\mathbf{E}$ and the fibre strain $e$. 
If a set of $\mathbf{n}$ identical and uncoupled fibres were involved in the impact process, the total force exerted on the projectile would be calculated by multiplying the number of fibres by the force obtained from Eq. (2). Then the projectile motion would be computed by integrating the differential equation given by Newton's second law. That is:

$$
-2\left(\frac{\mathbf{E} \mathbf{S}_{\mathbf{f}} \mathbf{n}}{\mathbf{m}_{\mathrm{p}}}\right) \frac{\mathbf{e z}}{\mathbf{l}}=\frac{\mathrm{d}^{2} \mathbf{z}}{\mathrm{dt}^{2}}
$$

where $\mathbf{m}_{\mathrm{p}}$ is the projectile mass, and $\mathbf{z}$ and $\mathbf{l}$ are the distance moved by the projectile and the actual fibre length affected at time $\mathbf{t}$ by the transverse wave propagation, respectively (see Figure 1). If the mass of the moving fibre in contact with the projectile tip becomes significant in relation to that of the projectile, Eq. (3) would have to be modified by adding the mass $\mathbf{m}_{\mathbf{f}}$ of the fibres in front of the penetrator to the projectile mass $\mathbf{m}_{\mathbf{p}}$.

For the case of a rigid cylindrical blunt projectile of radius $\mathbf{R}$ some changes should be introduced in the above equations. When such a projectile impacts a set of parallel identical fibres, each of them is directly impacted along a length which depends on the position relative to the centre of the projectile base. So, the fibre that coincides with the base diameter is impacted over a length larger than that impacted by the projectile edge. To include this in the analysis, a weighted value of the impacted fibre length is considered for all fibres. To simplify computations, the circular base of the projectile is substituted by a square of the same area and thus all the fibres behave in the same manner. The new so obtained motion equation would apply until a fibre failure criterion is verified. The most simplistic way to consider this is in terms of an ultimate breaking strain or stress criterion. It does not incorporate the great dependency of fibre rupture on parameters such as temperature and strain rate, which have been observed in polymeric materials. Although a criterion such as that proposed by Zhurkov [3] may be applicable, there is a lack of data about the parameters that it involves. In order to facilitate the use of the model herein proposed, the ultimate stress criterion is used, taking a breaking stress corresponding to a typical magnitude of the strain rate in these kind of problems.

\subsection{Model for an unidirectional composite subject to transverse impact}

When unidirectional composites are involved in impact problems, the outlined model may also be employed. It is well recognized (see figure 2 of Reference [4], for example) that ballistic efficiency of composite materials (expressed in terms of ballistic limit), diminishes when resin content gets higher. The effect of the resin may be explained from a double perspective: on one hand, the resin tends to restrain the transverse motion of the fibres; on the other hand, when the resin matrix has failed, it accompanies the fibres in their motion without collaborating in bearing any stresses and thus only adds mass to the fibres, decreasing the transverse wave velocity as well as the volume of composite moving after impact. For these reasons, Morrison and Bader [5] suggest to add the matrix mass to that of the fibres as a dead mass in order to incorporate such effects. Thus the above-described model may be utilized in impact problems involving unidirectional laminates if the following set of simplifying hypotheses are considered: all the plies behave in the same manner; $b$ ) the matrix breaks immediately after impact and $c$ ) the broken matrix moves together with the fibres only adding a dead mass to the former.

Another important aspect in this type of problem arises when projectile deformation is considered. As is known lead projectiles, for instance, deform plastically very much when impacting a multilayer composite panel. When the projectile suffers significant plastic deformation throughout the impact event the area of contact between projectile and fabric increases with time. The model does not incorporate the projectile deformation and thus this restriction should be kept in mind when applying the model to such situations.

To illustrate the model prediction capability, the problem of a $9 \mathrm{~mm}$ Parabellum projectile impacting against a 34 layer polyethylene fibre composite with an elastomeric matrix is analyzed. The composite is fabricated by DSM and its commercial name is Dyneema UD66. Following the manufacturer's information the fibre density is $970 \mathrm{~kg} / \mathrm{m}^{3}$, the areal density of a ply is about $0.166 \mathrm{~kg} / \mathrm{m}^{2}$, and the number of fibres per centimetre is 5.9. In Table 1 the results of the predictions are compared with some manufacturer's data [6], for the impact problem on a composite of areal density of $5 \mathrm{~kg} / \mathrm{m}^{2}$ at two projectile velocities below the ballistic limit. Predictions are made at each velocity for two different impacts, case A corresponding to a projectile radius of $4.5 \mathrm{~mm}$ (standard radius of the projectile), and case B for a radius of $6.0 \mathrm{~mm}$ (typical value of the radius of the considered projectile after impacting a composite target). 


\begin{tabular}{|c|c|c|c|}
\hline \multirow[b]{3}{*}{ Projectile velocity $(\mathrm{m} / \mathrm{s})$} & \multicolumn{2}{|c|}{ Table 1} & \multirow{3}{*}{$\underset{300}{\text { Experimentalvalue }}$ [6] } \\
\hline & Case $\mathbf{A}$ & Case B & \\
\hline & $300 \quad 420$ & $300 \quad 420$ & \\
\hline Trauma (mm) & $29.3 \quad 33.1$ & $24.4 \quad 27.4$ & $23.0 \quad 31.1$ \\
\hline Time $(\mu s)$ & 195 & $206 \quad 167$ & \\
\hline Maximum strain & 2.6 & 1.62 .6 & \\
\hline Affected zone radius (mm) & $63.4 \quad 63.0$ & $58.3 \quad 57.8$ & \\
\hline
\end{tabular}

Some consequences should be commented upon. The first aspect concerns the low influence of the radius of the projectile on the maximum strain recorded during the modelling calculation. This suggests that this maximum strain is governed by other factors such as projectile impact velocity, elastic fibre properties, etc. instead of the projectile radius. However, trauma (In ballistic applications this term is used for the maximum displacement of the fabric along the projectile axis direction [6]. It should not be misunderstood with its synonym for medical reactions) seems to be strongly dependent on the projectile diameter. Normally, low values of this parameter (undeformed projectile hypothesis) lead to higher traumas than when greater projectile dimensions due to plastic deformation are considered. This fact suggests that the greater the plastic deformation of the projectile the smaller the trauma.

It was also analyzed the problem of a $7.62 * 51$ Ball projectile at $820 \mathrm{~m} / \mathrm{s}$ impacting 150 plies of a Dyneema UD 66 composite $25 \mathrm{~mm}$ thick. Experimentally the projectile is defeated by the composite armour. In applying the engineering model it was necessary to assume a maximum breaking stress corresponding to a strain in the composite about $6.46 \%$, which greatly exceeds the maximum static strain that this material can support although dynamic tests carried out using a Hopkinson Bar device lead to values of the critical strain close to $5 \%$.

\subsection{Model for a woven fabric subject to transverse impact}

Ballistic impact on fabrics has been studied by many authors (several references about this subject may be found in Navarro [7]). Photographs of fabric panels during and after the impact event show that impacted fabrics deform to conical shape, in a similar manner to the deformation of a single fibre. This suggests the possibility of employing the above analytical model to this problem also. However, the direct application of such model is not possible, because a set of new phenomenological aspects appears. In fact, the fibres of the fabric now interact with each other, and wave propagation in the fibres is now affected by the presence of the filling fibres. Reflection and refraction processes are involved, and thus strain distribution along the fibre differs from that of a single fibre situation. The numerical and engineering models for this problem should be more sophisticated and should involve the interaction parameters that characterize the warp-filling fibre interaction.

Direct analyses of the problem using the finite element method have been carried out by several authors (see several references in [7]). When the fabric is very dense, modelling the fabric as a continuum may be a good solution. The problem arises in deciding whether the fabric can be modelled in such a way or must be modelled taking into account the physical presence of fibre joints, and thus the directional property changes. One of the most important works on this topic is that of Roylance et al. [8], who extended a dynamic finite-element technique, previously developed for single fibres, to model woven panels. This shows that a woven textile panel exhibits substantial differences compared to the equivalent impact on a single fibre, because of the complex interactions due to the fibres crossovers. At each node of the finite element mesh, a portion of the onward-propagating tensile strain wave is reflected, attenuating the magnitude of the strain at the wave front, while increasing the strain level of fibres placed in the neighbourhood of the projectile. Another important consequence of the works of Roylance and co-workers is the fact that the vast majority of ballistic energy was seen to be stored in the orthogonal fibres passing through the impact point, while the other fibres were essentially ineffective. Also they observed that the kinetic energy acquired by the fibres just in contact with the projectile was concentrated only in such fibres and that it was of the same order of magnitude as the strain energy of those fibres. They assumed a linear elastic model for fibre behaviour and a Zhurkov's failure criterion [3]. Another contribution of Roylance [9] to the study of wave propagation in woven panels is the solution, by means of the finite differences method, of the energy wave transmission at crossovers, considering the slippage between fibres.

To account for the influence of transverse fibres in the present model, the fabric pattern depicted in Figure 2 , in which the zone of the fabric affected by the transverse wave is circular, is assumed. From geometrical considerations, the strain in the transverse fibres can be computed and thus the resistive force exerted by them. According to Roylance's observations, only the kinetic energy developed in the fibres passing through the projectile base was considered. The interaction between warp and filling fibres is incorporated 
into the model assuming a Coulomb frictional effect and thus considering the possibility of slippage between such fibres.

A failure criterion based on a critical force that fibres are able to resist is assumed instead of considering a critical strain. The reason for this is based on Hopkinson Bar test involving fabrics: the fibre strain at failure shows a great deal of dispersion while this does not occur with the maximum force.

To illustrate the model prediction capabilities, the problem of a projectile impacting at $400 \mathrm{~m} / \mathrm{s}$ against 27 plies of kevlar $\mathrm{Ht}\left(190 \mathrm{~g} / \mathrm{m}^{2}\right.$ per ply) is considered. In this problem the projectile was defeated by the fabric panel although about a third of the plies broke. From tribological tests, a dynamic friction coefficient of 0.25 between kevlar fibres was assumed. Based on Hopkinson Bar test results, a value of the maximum force per centimeter of about $1310 \mathrm{~N}$ at a strain rate of $1000 \mathrm{~s}^{-1}$ was assumed in the analysis. The experimental value of the trauma was about 35 $\mathrm{mm}$ and the radius of the zone affected by the transverse wave, where a clear delamination was observed, ranged from 23 to $38 \mathrm{~mm}$. The computed results using the model are given in Figure 3 , in which time histories of the plate deflection (Fig. 3a), the radius of the zone affected by the transverse wave (Fig. 3b), the fibre strain (Fig. 3c) and the maximum force developed by all the fibres in the longitudinal direction (Fig. 3d), are drawn.

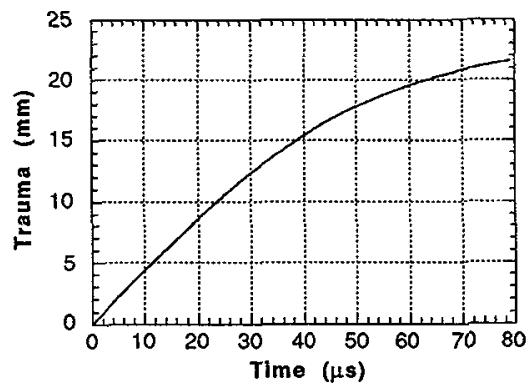

Figure $3 \mathrm{a}$

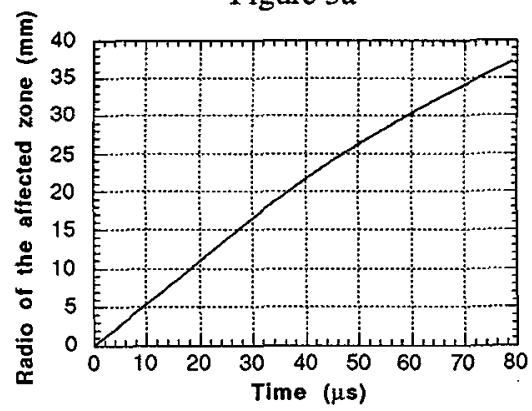

Figure $3 b$

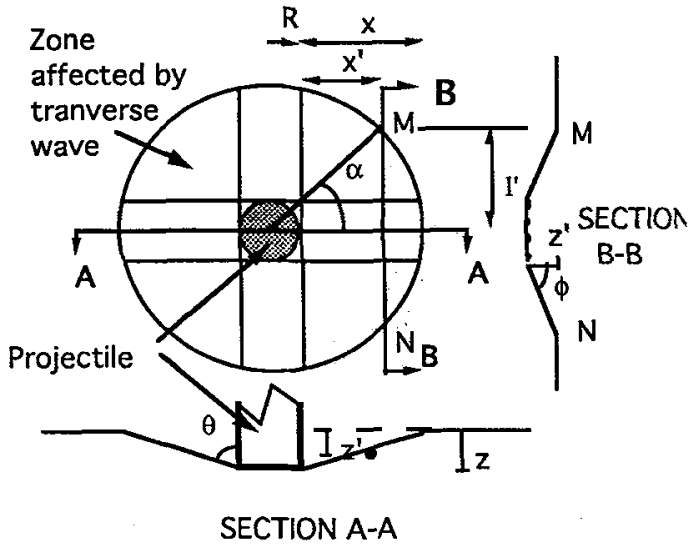

Figure 2

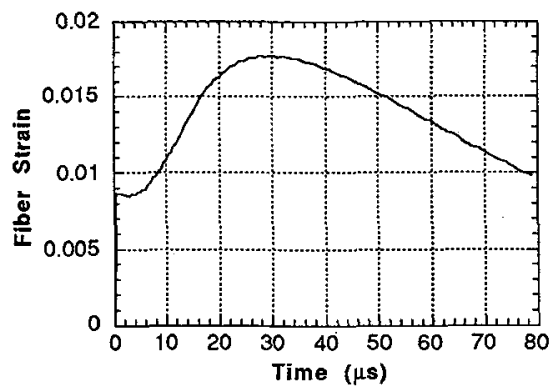

Figure $3 \mathrm{c}$

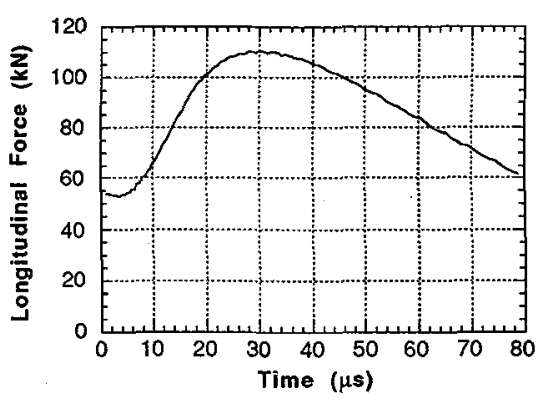

Figure $3 d$

\subsection{Model for a fabric reinforced composite subject to transverse impact}

The above described model may be applied to problems in which a composite made of fabric and resin is involved. The fabric behaviour can be modelled incorporating the effect of resin content in the same 
manner as for the unidirectional composite model: that is, the effect of the resin has only an inertial character. To show the prediction capabilities of the simplified model, another impact problem is analyzed. This problem corresponds to the impact of a $12.7 \mathrm{~mm}$ diameter hard-steel cylindrical bullet impacting a 10 ply laminated Kevlar 29/polyester. The impact velocity was $124 \mathrm{~m} / \mathrm{s}$, and the areal density of the laminate was $4.91 \mathrm{~kg} / \mathrm{m}^{2}$. Other parameters of kevlar and polyester matrix were taken from the open literature.

In the problem considered the projectile perforates the composite plate and there are available experimental measurements [10] of the displacement of the composite plate along the projectile axis direction, the projectile velocity and the defeating force. Figure 4 shows a comparison between the experimental measurements and computed results of the above-mentioned parameters. There is a large degree of agreement which illustrates the model prediction capabilities. In another paper published elsewhere [11] the model described here is used, in conjunction with another simplified model for ceramics, to study the behaviour of mixed ceramic/composite targets under impact conditions.

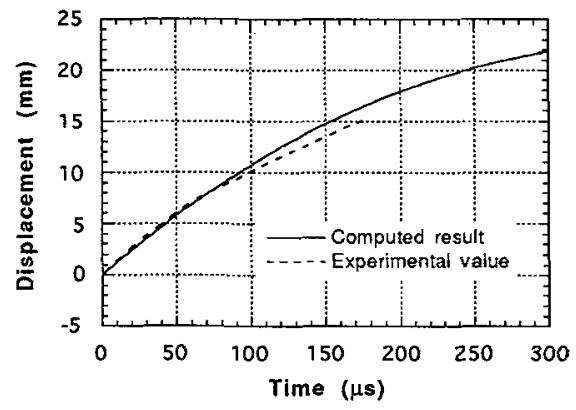

Figure 4a

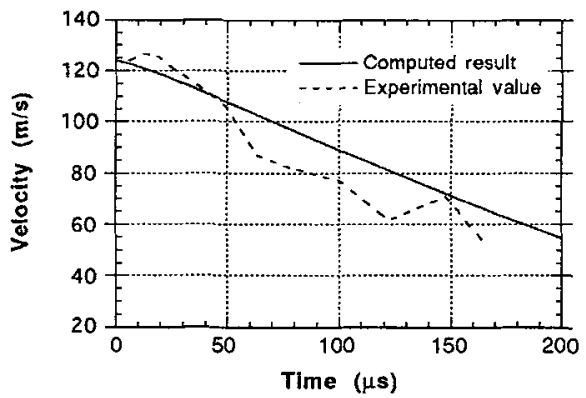

Figure $4 b$

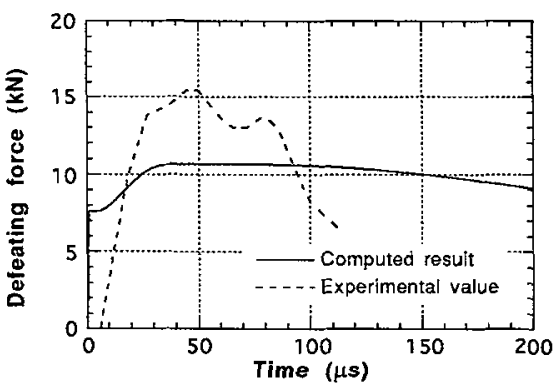

Figure $4 c$

\section{CONCLUSIONS}

Although the model is based on rough hypotheses, in particular with regard to the effect of the matrix resin, the filling-warp interaction, projectile undeformability, etc., the results it gives are in agreement with experimental observations. This simplified tool needs a very small computer time, which allows many parametric analyses to be undertaken. Further effort should be made to incorporate in the model the matrix influence and thus the ply-ply interaction.

Ackowledgements: The authors are indebted to the Comisión Interministerial de Ciencia y Tecnología of Spain for its financial support of this research (Project: MAT92-1022-C02-02).

\section{REFERENCES}

1 Smith, J.C., et al.,Textile Research Journal, 30, No. 10, (1960) 752-760.

2 Petterson, D.R., et al., Textile Research Journal, 30, No. 6, 411-421.

3 Zhurkov, S.N., Int. J. Fracture Mechanics,1,311 (1965).

4 Dupont de Nemours Int.," Lightweight protective armour of Kevlar ${ }^{\circledR}$ Aramid", (1983).

5 Morrison, C.E. and Bader, M.G., First ECCM, Bordeaux, (1985) 706-712.

6 DSM, " Dyneema Lightweight and personal armour", The Netherlands, 1990.

7 Navarro, C., Proc. ICCM/9, Vol. I, (1993) 131-138.

8 Roylance, D., Textile Research Journal, 47, (1977) 34-41.

9 Roylance, D., Fibre Science and Technology, 13, (1980) 1068-1072.

10 Zhu,G., Int. J. Solids Struct., 29, No. 4, (1992) 421-436.

11 Zaera, R., et al., Proc. SUSI'94, (1994). 